PR-16

\title{
NUCLEOPHILIC SUBSTITUTION OF NITRO GROUP IN DIHYDROAZOLE[5,1-C][1,2,4]TRIAZINES
}

\author{
R. A. Drokin, D. V. Tufyakov, E. K. Voinkov, E. N. Ulomsky, V. L. Rusinov \\ Ural Federal University of the first President of Russia B. N. Yeltsin. 19 Mira St.,. \\ Yekaterinburg, 620002, Russia. \\ E-mail: drokinroman@gmail.com
}

\begin{abstract}
Previously, nucleophilic substitution of a nitro group in a series of azoloazines by O-, S-, Nnucleophiles was studied [1-3], but works describing the substitution of a nitro group in dihydroazolo $[5,1-c][1,2,4]$ triazines by primary aromatic amines are absent due to the lack of nucleophilicity of the latter.
\end{abstract}

We managed to carry out the nucleophilic substitution of the nitro group into fragments methoxyaniline by boiling in acetonitrile.

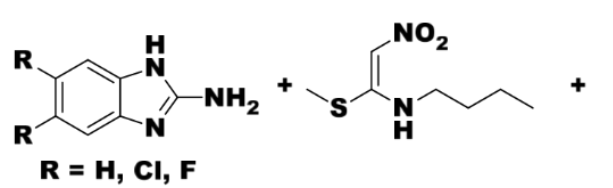

(1)

(2)

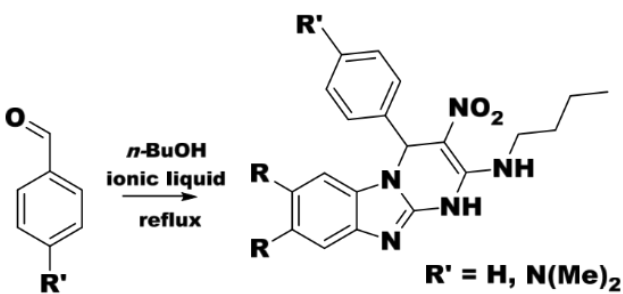

(4)

The reactions of dihydroazolo[5,1-c][1,2,4]triazines with hydrazine hydrate were previously studied. The authors suggested that heating nitroazolotriazine in ethanol with hydrazine hydrate leads to the reductive decomposition of the triazine ring to form 3-methyl-5-(N-methylamino)-1,2,4-triazole [3].

We found that nitrodihydroazolo[5,1-c][1,2,4]triazines react with dimethylmethylenehydrazine to produce the nucleophilic substitution product at low temperatures.<smiles>[R]c1nc2n(C)nc(NN)c(=O)n2n1</smiles>

\section{References}

1. Beck J.R. // Tetrahedron. - 1978. - Vol. 34. - P. 2057

2. Rusinov, V. L., Sapozhnikova, I. M., Ulomskii, E. N., Medvedeva, N. R., Egorov, V. V., Kiselev, O. I., . . Chupakhin, O. N. (2015). Nucleophilic substitution of nitro group in nitrotriazolotriazines as a model of potential interaction with cysteinecontaining proteins. Chemistry of Heterocyclic Compounds, 51(3), 275-280

3. Nitroazines. 9. Characteristic features of nucleophilic substitution of the nitro group in dihydroazolo[5,1-c] [1,2,4]triazines/ V. L. Rusinov, E. N. Ulomskii, O. N. Chupakhin [et al.]// Chemistry of Heterocyclic Compounds. - 1989. Vol. 34 . - № 2 C. 209-213 